Revue d'histoire de l'Amérique française

REYUE D.HISTOIRE DE L'AMÉRIQUE FRANÇAISE

\title{
CASTONGUAY, Stéphane et Camille LIMOGES, François Blanchet, 1 : l'étudiant et le savant (Montréal, VLB éditeur, 2004), 399 p.
}

\section{Rénald Lessard et Stéphanie Tésio}

Volume 59, numéro 1-2, été-automne 2005

URI : https://id.erudit.org/iderudit/012727ar

DOI : https://doi.org/10.7202/012727ar

Aller au sommaire du numéro

Éditeur(s)

Institut d'histoire de l'Amérique française

ISSN

0035-2357 (imprimé)

1492-1383 (numérique)

Découvrir la revue

Citer ce compte rendu

Lessard, R. \& Tésio, S. (2005). Compte rendu de [CASTONGUAY, Stéphane et Camille LIMOGES, François Blanchet, 1 : l'étudiant et le savant (Montréal, VLB éditeur, 2004), 399 p.] Revue d'histoire de l'Amérique française, 59(1-2), 129-131. https://doi.org/10.7202/012727ar d'utilisation que vous pouvez consulter en ligne.

https://apropos.erudit.org/fr/usagers/politique-dutilisation/ 
précédents s'étaient en effet contentés d'intuitions sur la paternité du document, alors que Brandão scrute plus attentivement son histoire afin d'en dater la rédaction et de déceler avec plus de certitude l'identité de l'auteur. Le résultat est une argumentation étoffée de l'hypothèse émise par A. Boisvert, qui avait attribué le document à René Cuillerier, un habitant de Lachine capturé par les Iroquois en 1661 et qui passa un an et demi en captivité chez les Onéiouts. La qualité de la transcription est aussi nettement supérieure à celle proposée par A. Boisvert, qui avait pris le parti de moderniser la graphie originale; la minutie de Brandão lui permet même de corriger, au passage, quelques «coquilles» oubliées par les éditeurs de Recherches amérindiennes au Québec.

On ne peut que saluer le caractère scientifique de l'annotation ajoutée au texte par Brandão, qui présente les études ethnographiques les plus récentes sur la culture iroquoise. Cette attitude tranche radicalement avec celle d'A. Boisvert, dont les quelque 191 notes explicatives, souvent plus lourdes que pertinentes, projettent une vision extrêmement archaïque de la société iroquoise, décrite comme déraisonnable, «inhumaine» et «sanguinaire». Seulement, comparée à ces 191 commentaires, l'annotation de Brandão paraît peu ambitieuse et l'on aurait souhaité qu'une nouvelle édition du document fasse une mise en parallèle plus systématique avec d'autres sources et propose davantage de références bibliographiques. Ou peut-être aurait-il été plus judicieux de publier un recueil colligeant plus d'un document sur les mœurs iroquoises?

MAXIME GOHIER

Département d'histoire Université du Québec à Montréal

CASTONGUAY, Stéphane et Camille LIMOGES, François Blanchet, 1: l'étudiant et le savant (Montréal,VLB éditeur, 2004), 399 p.

À l'ombre des grands noms de la médecine et des sciences en Europe, en Amérique du Nord et au Canada, les deux auteurs chevronnés, Stéphane Castonguay et Camille Limoges, nous font connaître, dans ce premier tome, une de ces figures canadiennes souvent ignorées, délaissées ou mal perçues par l'historiographie canadienne. Homme politique célèbre, membre du Parti patriote, éminent médecin de la bourgeoisie québécoise, François Blanchet (1776-1830) a participé à cette vive émulation scientifique au tournant des XviII ${ }^{e}$ et XIX ${ }^{e}$ siècles. 
À cet effet, leur ouvrage propose deux parties dans lesquelles nous pouvons retenir trois sections essentielles qui rythment la vie de ce médecin bas-canadien, à l'excellente réputation professionnelle. La première décrit ses années d'études dans les villes de Québec et de New York; la seconde s'attarde sur la doctrine scientifique de ce médecin (c'est-à-dire sur sa conception de la médecine et des théories médicales en vigueur à son époque); enfin la troisième partie est une reproduction de son ouvrage qui s'intitule Les Recherches sur la médecine ou l'application de la chimie à le médecine, publié en juillet 1800 . Les deux premières sections proposent une mise en contexte très détaillée, excellente et claire au sujet des théories qui jalonnent l'histoire de la médecine et l'histoire des sciences. En effet, à cette époque, les diverses disciplines scientifiques ne sont pas séparées comme à l'heure actuelle, et bien souvent les savants, depuis au moins l'époque médiévale, sont ce que nous nommons aujourd'hui des multidisciplinaires. Cette mise en contexte constitue, sans doute, l'apport le plus original de cet ouvrage. D'ailleurs, l'appareil scientifique est des plus élaborés, avec plus de 140 pages de notes (806 notes dont 306 sur le manuscrit) et de références bibliographiques, avec un index détaillé qui complète le tout.

Issu d'une famille canadienne-française, François Blanchet naît le 3 avril 1776 à Saint-Pierre-de-la-Rivière-du-Sud, près de l'actuel Montmagny. Ce fils d'agriculteur aisé commence ses études au séminaire de Québec à partir de 1790-1791, qu'il quitte en janvier 1794 afin de se consacrer à un apprentissage en médecine jusqu'en 1799, auprès de James Fisher, chirurgien militaire, membre de l'élite médicale de Québec. En effet, la médecine au Canada, surtout après la Conquête en 1760, s'apprend par le système de l'apprentissage - le Canada ne bénéficiant pas d'écoles, d'instituts ou d'universités comme son pays voisin, les États-Unis - ou bien les jeunes gens se rendent en Écosse à l'Université d'Édimbourg. François Blanchet se perfectionne à New York au Columbia College dès 1799. Les auteurs expliquent fort bien, à cet effet, tout le système de l'enseignement de la médecine du xviII ${ }^{\mathrm{e}}$ siècle en Amérique du Nord, tant au Canada qu'aux États-Unis, ainsi que son contenu, très théorique, qui correspond aux exigences du moment.

En cette fin du XviII ${ }^{e}$ siècle et en ce début du XIX ${ }^{e}$ siècle, François Blanchet s'inscrit à la charnière de deux mouvements théoriques scientifiques qui marquent l'Europe et l'Amérique: d'un côté, la persistance de la théorie humorale d'Hippocrate et de Galien et le développement du vitalisme de Stahl, et de l'autre, la naissance et le développement de la 
médecine clinique, avec le rôle primordial, et sans précédent, de l'école française, rôle qui perdure pendant une bonne partie du $\mathrm{xIx}^{\mathrm{e}}$ siècle. C'est la fin d'une tradition médicale ancienne qui laisse la place à la médecine moderne. C'est à New York que François Blanchet commence à rédiger des articles médicaux sur les préoccupations du moment, c'est-à-dire le rôle de la chimie dans la médecine. Il faut dire qu'un Antoine Laurent de Lavoisier en France, que la Révolution française s'est chargée de raccourcir en 1794, a œuvré en ce sens et a eu une grande influence sur ses contemporains et successeurs: père de la chimie moderne et également physicien à ses heures, on lui doit, entre autres, la nomenclature chimique (les symboles chimiques), la composition de l'air et de l'eau, la découverte du rôle de l'oxygène dans les combustions et dans la digestion animale. Certes, l'écrit de François Blanchet ne fait pas autorité à l'époque parmi ses confrères européens et nord-américains, mais il a le mérite de poser une des premières pierres de l'édifice qui s'appelle «la médecine moderne au Canada». C'est aussi une façon pour les deux auteurs de montrer qu'au-delà de la très forte influence médicale et scientifique de l'Europe, surtout de la France, et de l'influence certaine des États-Unis sur Blanchet, le Canada peut aussi jouer un rôle international, même si les résultats ne sont pas à la hauteur des attentes.

Un dernier point mérite d'être souligné: le courage de l'éditeur de publier sur papier des éditions de textes anciens et des versions annotées de documents significatifs. Après plus d'un quart de siècle d'abandon relatif, ce type de travail redevient populaire grâce à Internet. Mais des chercheurs, comme Stéphane Castonguay, Camille Limoges, Georges Aubin ou France Martineau, redonnent aujourd'hui leurs lettres de noblesse aux éditions sur papier.

Dans le deuxième tome, les auteurs proposent d'explorer la carrière postérieure de médecin et d'homme politique de François Blanchet. Si la qualité de la recherche et de l'analyse se maintient, nous sommes en droit d'espérer un nouvel ouvrage de qualité. 